GOLDBERG (Hallamshire Hospital Medical School, Sheffield) Immunological and immunochemical parameters are useful adjuncts to the more usual liver function tests in the diagnosis and management of hepatobiliary disease. The profile, as it has been developed, consists of a series of tests which are technically easy to perform.

Serum immunoglobulin levels in liver disease are of particular value in distinguishing between the inflammatory and the essentially surgical hepatic lesions. Recognizable patterns of immunoglobulin profile may be discerned. This pattern recognition is improved by the consideration of the non-organ specific antitissue antibodies to nuclei, mitochondria, and smooth muscle. The detection of $\alpha_{1}$ fetoprotein by a purely qualitative procedure assists in the diagnosis of increased hepatocyte turnover. The $\alpha_{1}$ antitrypsin level is determined in selected cases, mainly paediatric, to exclude the intrahepatic cholestasis and progressive cirrhosis of antitrypsin deficiency. The profile is completed by screening for HAA and its attendant antibody.

The immunochemical determination of albumin has been added to the profile. This was done because the immunochemical albumin represents a truer picture of the serum albumin than the more conventional methods in those cases where the level is abnormally low, or where an elevated bilirubin interferes with its determination.

Pattern recognition with this profile may be summarized with reference to seven main conditions or groups of conditions:

\section{Acute hepatitis}

IgM elevation $300-1000 \mathrm{mg} / 100 \mathrm{ml}$ (345$115000 \mathrm{IU} / \mathrm{ml}$ ) in $70 \%$ and antibody to smooth muscle in $60 \%$. In the late stages of the disease IgG elevation become more prominent, and weak positive reactions for $\alpha_{1}$ fetoprotein are seen with hepatocyte regeneration.

\section{Chronic hepatitis}

IgG and IgM elevations in $80 \%$, antibodies to nuclei in $45 \%$, and to smooth muscle in $54 \%$. This group may be subdivided into chronic aggressive hepatitis in which IgG elevation and antibodies to nuclei predominate, and chronic persistent hepatitis in which IgG and IgM elevation and antibodies to smooth muscle predominate.

\section{Primary biliary cirrhosis}

IgM elevation $350-1500 \mathrm{mg} / 100 \mathrm{ml}$
(400-172 $500 \mathrm{IU} / \mathrm{ml})$ in $100 \%$ and antibody to mitochondria in $85 \%$. Moderate elevations of IgG were seen in $60 \%$.

\section{Micronodular cirrhosis}

IgA elevations in excess of $600 \mathrm{mg} / 100 \mathrm{ml}$ $(350 \mathrm{IU} / \mathrm{ml})$ were seen in $70 \%$.

\section{Cholecystitis}

Immunoglobulins and antibodies usually normal although four/eight cases of cholangitis showed elevation of IgM with attendant mitochondrial antibodies.

\section{Extrahepatic obstruction}

Immunoglobulins normal except for a variable mild IgA elevation. Antibodies uniformly negative.

\section{Intrahepatic cholestasis}

Immunoglobulins and antibodies normal save for two individuals who had IgM elevations and antibodies to nuclei.

\section{Histological Aspects of Jaundice}

P. J. SCHEUER (Department of Histopathology, Royal Free Hospital, London) Histological examination of liver biopsies helps to distinguish between different causes of jaundice. Haemolysis leads to iron deposition in the liver. In Gilbert's disease, a common form of non-haemolytic hyperbilirubinaemia, the liver is histologically normal, whereas in the Dubin-Johnson type of conjugated hyperbilirubinaemia the liver cells contain abundant iron-negative pigment.

The above conditions are distinct from cholestasis, in which bile as a whole fails to reach the duodenum in normal quantities, either because of mechanical obstruction or as a result of intrahepatic disease. From the histological point of view, cholestasis is the presence of demonstrable bile in tissue sections.

In acute cholestatic jaundice the practical problem in liver biopsies is usually the differentiation of acute hepatitis of viral type, drug-induced cholestasis such as that produced by chlorpromazine, and mechanical obstruction to large bile ducts. Other possibilities include hepatitis due to other causes such as drugs, alcohol, or infective agents, and chronic liver disease presenting acutely. In viral hepatitis the diagnostic lesion is the widespread and pleomorphic alteration of liver cells, accompanied by inflammatorycell infiltration. Portal lesions are present but are less specific. Some drugs may produce an identical picture. Drug- induced cholestasis is accompanied by little inflammation, and may be very difficult to distinguish from other forms of cholestasis without inflammation, such as idiopathic recurrent cholestasis and cases of mechanical biliary obstruction in which typical portal changes have failed to develop. These changes include oedema and acute inflammation of the tracts and proliferation of bile ducts.

Of the many forms of chronic cholestasis, primary biliary cirrhosis may be more common than previously supposed. Needle biopsies may not contain the classical bile duct lesions and granulomas, ? but may show characteristic changes, i notably lymphoid aggregates.

\section{Bilirubin Conjugation and Excretion}

\section{के}

BARBARA H. BILLING (Department of $\stackrel{\circ}{2}$ Medicine, Royal Free Hospital, London) $\overrightarrow{-}$ Bilirubin can only be excreted in the bile (or urine) if it is first converted into a $\underset{D}{\vec{D}}$ polar derivative. This is mainly achieved by the formation of mono and di-glucuronide esters although recent studies have : indicated that conjugation with glycosides and acidic disaccharides may also be important, particularly in obstructive jaundice. The microsomal enzyme responsible for the conjugation of bilirubin, bilirubin UDP glucuronyl transferase, appears to be the rate-limiting factor in the transfer of bilirubin from the plasma into the bile. A deficiency of the enzyme will therefore reduce the rate of clearance of bilirubin from the plasma and cause an unconjugated hyperbilirubinaemia, such as is commonly seen in the newborn. In the adult an absolute deficiency of the enzyme is extremely rare (Crigler Najjer syndrome) but a relative deficiency is not uncommon and has been found in all patients with Gilbert's syndrome. This benign condition is characterized by a $\frac{7}{0}$ mild unconjugated hyperbilirubinaemia $(0.8-5.0 \mathrm{mg} / 100 \mathrm{ml})$ in the absence of overt $\mathrm{N}$ haemolysis or coexistent hepatic or systemic disease. The incidence of Gilbert's syndrome in the general population is $\mathrm{W}$ uncertain but values of the order of $0.5 \%$ for males and $0.15 \%$ for females have been cited. The degree of hyperbilirubinaemia tends to fluctuate and can be reduced by the administration of drugs such as phenobarbitone and glutethimide and raised by fasting and infection.

The capacity of the liver to conjugate and thus to excrete bilirubin is rarely $\stackrel{\mathbb{D}}{\square}$ exceeded except after a severe haemolytic $\underset{-}{\sigma}$ episode or following the administration of 